\title{
Challenges in Diabetic Macular Edema Management: An Expert Consensus Report
}

\author{
Patricia Udaondo (ID) \\ Alfredo Adan (D) ${ }^{2}$ \\ Luis Arias-Barquet (iD ${ }^{3}$ \\ Francisco J Ascaso 4 \\ Francisco Cabrera-López (iD) 5 \\ Verónica Castro-Navarro ${ }^{6}$ \\ Juan Donate-López ${ }^{7}$ \\ Alfredo García-Layana ${ }^{8}$ \\ Francisco Javier Lavid (1D ${ }^{9}$ \\ Mariano Rodríguez-Maqueda ${ }^{10}$ \\ José María Ruiz-Moreno" \\ 'Department of Ophthalmology, Hospital \\ Universitario y Politecnico la FE, Valencia, \\ Spain; ${ }^{2}$ Department of Ophthalmology, \\ Hospital Clinic Barcelona, Barcelona, \\ Spain; ${ }^{3}$ Department of Ophthalmology, \\ University Complex Bellvitge, Barcelona, \\ Spain; ${ }^{4}$ Department of Ophthalmology, \\ Hospital Universitario Lozano Blesa, \\ Zaragoza, Spain; ${ }^{5}$ Department of \\ Ophthalmology, Hospital Universitario \\ Insular, Las Palmas de Gran Canaria, \\ Spain; ${ }^{6}$ Department of Ophthalmology, \\ Hospital General Universitario, Valencia, \\ Spain; ${ }^{7}$ Department of Ophthalmology, \\ Hospital Clínico San Carlos, Madrid, \\ Spain; ${ }^{8}$ Department of Ophthalmology, \\ Clínica Universitaria de Navarra, \\ Pamplona, Spain; 'Department of \\ Ophthalmology, Hospital Punta Europa, \\ Algeciras, Cádiz, Spain; ${ }^{10}$ Department of \\ Ophthalmology, Hospital Virgen del \\ Rocío, Sevilla, Spain; " 'Department of \\ Ophthalmology, Hospital Puerta de \\ Hierro, Majadahonda, Madrid, Spain
}

Correspondence: Patricia Udaondo Aiken Prevención y Cirugía ocular, Pizarro, 15 Bajo, Valencia, 46004, Spain Tel +34647869228

Email draudaondo@gmail.com
Purpose: This paper aimed to present daily-practice recommendations for the management of diabetic macular edema (DME) patients based on available scientific evidence and the clinical experience of the consensus panel.

Methods: A group of Spanish retina experts agreed to discuss different aspects related with the clinical management of DME patients.

Results: Panel was mainly focused on therapeutic objectives in DME management; definition terms; and role of biomarkers as prognostic and predictive factors to intravitreal treatment response. The panel recommends to start DME treatment as soon as possible in those eyes with a visual acuity less than 20/25 (always according to the retina unit capacity). Naïve patient was defined, in a strict manner, as a patient who, up to that moment, had never received any treatment. A refractory DME patient may be defined as the one who did not achieve a complete resolution of the disease, regardless of the treatment administered. Different optical coherence tomography biomarkers, such as disorganization of the retinal inner layers, hyperreflective dots, and cysts, have been identified as prognostic factors.

Conclusion: This document has sought to lay down a set of recommendations and to identify key issues that may be useful for the daily management of DME patients.

Keywords: diabetes, diabetic macular edema, optical coherence tomography, inflammation, biomarkers, consensus

\section{Introduction}

As the prevalence of diabetes mellitus is rising up, the importance of diabetic eye disorders increases. ${ }^{1,2}$ In Europe, it was estimated that approximately 6.4 million people are currently affected by any diabetic eye disease and 8.6 million people will be affected in $2050 .^{3}$ In the year 2020, moderate-to-severe visual impairment due to diabetic retinopathy has been estimated in 4.06\% (Western Europe); 4.77\% (AsiaPacific, high income); and 4.99\% (United States, high income). ${ }^{2}$

Diabetic macular edema (DME) is a prevalent condition that impacts central visual acuity (VA), and, therefore, critically influence on patient's quality of life. ${ }^{4,5}$

The prevalence of DME in Europe was estimated in $3.7 \%$ and its pooled mean annual incidence in type-2-diabetes patients was $0.4 \%{ }^{3}$

The changes in the paradigm of DME treatment ${ }^{6-11}$ as well as the development of technological advances for diagnosis ${ }^{12-15}$ makes, from our point of view, necessary to reconsider the approach to the daily practice management of these patients.

The aim of this manuscript is to evaluate and respond to different issues related to the management of DME patients and to establish consensus-based recommendations to provide the ophthalmologists responsible for the management of diabetic 
patients with a frame of reference based on available scientific evidence and the clinical experience of the group.

\section{Methods}

A group of ophthalmologists in charge of Retina Units from 11 Spanish hospitals, working in collaboration, has developed a consensus report about different issues related to clinical management of patients with DME.

In the first meeting, performed on 13 February 2019, the panel of retina experts selected and agreed a first list of topics related to the clinical management of DME patients. The different subjects that focused the panel's attention were as follows: 1) Therapeutic objectives in DME management; 2) Definition of naïve, refractory, and chronic DME; and 3) Role of biomarkers as prognostic and predictive factors to intravitreal treatment response.

Attending to these subjects, the expert panel developed a list of questions. These questions were discussed, updated literature was reviewed and responses were agreed in different meetings held from February to October 2019 (six meetings in total).

A PubMed literature search for English, French, and Spanish language articles published to date was performed using the terms "Diabetic macular edema" AND "Treatment" OR "Diagnosis" OR "Biomarkers" OR "Management" OR "Outcomes". References cited in selected articles were also reviewed to identify additional relevant reports. Likewise, published national and international guidelines were also scrutinized.

An initial document was drafted and it was reviewed by all members of the panel of experts, who had the opportunity to make all the changes/suggestions/comments deemed necessary. Finally, after making the required revisions based on the panel feedback and reached a consensus, the final text was then validated.

\section{Results}

\section{Basic Concepts}

\section{Therapeutic Objectives in DME}

Functional and anatomic outcomes are usually selected as therapeutic objectives. ${ }^{16-34}$ While therapeutic objectives have been clearly defined in clinical trials, the identical criteria may not be applicable to routine clinical practice. Sometimes the objective of DME treatment is reduced to maintain VA. However, our treatment goals with DME patients should be more ambitious than that with neovascular age-related macular degeneration patients. There is some evidence suggesting that functional response critically depends on baseline VA, because patients with good visual acuity have a ceiling effect with limited visual improvement, and the status of retina as there are patients with retinal damage without any possibility of functional improvement and the stabilization of vision avoid the vision loss is an achievement. ${ }^{20,21,35}$ Thus, our therapeutic objective in terms of vision should be:

- In patients with VA $20 / 25$ or better: observation and start to treat if vision worsens.

- In patients with high baseline VA (20/40 to 20/32): To maintain the VA.

- Although many clinicians initiate vascular endothelial growth factor inhibitors (anti-VEGF) treatment in DME patients when visual acuity is minimally affected or unaffected, Protocol $\mathrm{V}^{35}$ found that, in eyes with central involved DME and good vision, visual acuity remained stable, at least, during 2 years. That is why, our main goal is maintaining VA and, as a secondary objective, to improve it, if possible.

- In patients with low baseline VA (20/50 to 20/320): To improve the VA.

On the other hand, and since functional response depends on the status of the retina, ${ }^{36-41}$ the efficacy of a treatment cannot be measured just attending to functional response. It has been suggested that in eyes with DME, VA depends on retinal thickness ${ }^{42}$ and integrity of retinal structure (including inner and outer retina layer), especially the photoreceptor layer. ${ }^{43-45}$ Because the functional response depends on the anatomic status of the retina, from a daily practice perspective, the main therapeutic objective in DME patients should be to achieve the best anatomic response as fast as possible, and at that point we must check the maximum functional response the patient can get.

It should be taken into consideration that an early anatomic response may predict mid-to-long-term anatomic outcomes and, therefore, the functional ones. ${ }^{46}$

\section{When Patients Should Be Treated?}

The results of Protocol V, a prospective and randomized clinical trial that compared three different strategies, namely intravitreal aflibercept, focal/grid laser 
photocoagulation, or observation, suggested that in eyes with DME and good VA, aflibercept or laser photocoagulation appeared to be no superior to observation at 2 years. ${ }^{35}$

Moreover, the OBTAIN study, was as a 12-month, retrospective, multicenter, and observational cohort study conducted in a real-world setting, which included DME patients with baseline visual acuity $\geq 20 / 25$ Snellen and central subfield thickness $(\mathrm{CST})>250 \mu \mathrm{m} .{ }^{47}$ Among the 249 eyes included in the study, 94 eyes $(37.7 \%)$ did not receive any treatment during 12 months of follow-up, and 155 eyes $(62.2 \%)$ received treatment over the course of the study. Mean change in VA at the end of the study was $1.8 \pm 5.6$ letters and $-3.4 \pm 5.8$ letters in non-treated and in treated eyes, respectively. ${ }^{47}$ The results of this study suggested that most of the DME eyes with very good VA maintained that VA during the 12-month of follow up, whether the DME was treated or not. ${ }^{47}$

Although according to the results of the Protocol $\mathrm{V}^{35}$ observation until vision deterioration occurs seems to be a feasible approach in DME with good baseline VA, these findings may not be applicable to all eyes with DME with good VA. ${ }^{48}$ For example, mean CST was very low (311 $\mu \mathrm{m})$ as compared with previous DME clinical trials. This is clinically relevant, since eyes with CST $\geq 400 \mu \mathrm{m}$ may have a different treatment response than eyes with a thinner $\mathrm{CST}^{49}$

Real-life treatment patterns in newly diagnosed DME patients were evaluated by means of an analysis of the American Academy of Ophthalmology Intelligent Research in Sight (IRIS ${ }^{\circledR}$ ) Registry. ${ }^{50}$ A total of 13,410 treatment-naive DME patients were included in the analysis. The results of this study have found that the treatment patterns within 28 days of initial DME diagnosis were as follows: observation in 9990 (74.5\%) patients; anti-VEGF in 2086 (15.6\%); laser photocoagulation in $1133(8.4 \%)$; corticosteroids in $133(1.0 \%)$; and combined therapy in 68 $(0.5 \%)$ patients. ${ }^{50}$

In daily practice, DME treatment does not represent an emergency. Nevertheless, because the presence of subretinal/intraretinal fluid may negatively impact on functional outcomes, early treatment would be highly recommended. ${ }^{51}$

Panel recommendation:

- When visual acuity is less than 20/25 DME treatment should begin as soon as possible, according to the capacity of the Retina Unit. The best scenario is one where patients are treated on the same day of diagnosis. However, the best scenario is rarely the most frequent. Therefore, in those cases in which patients cannot be treated on the same day, treatment should be administered within 10-15 days.

\section{Definitions}

\section{What is DME?}

DME can be defined as a retinal thickening $(\geq 250 \mu \mathrm{m})$ within one disk diameter of the center of the macula or definite hard exudates in this region. ${ }^{16}$

If fovea is involved, we speak about "Center-involved DME". ${ }^{16}$

Eyes with central macular thickness $(\mathrm{CMT}) \geq 500 \mu \mathrm{m}$, hard exudates within $500 \mu \mathrm{m}$ of the center of the macula with adjacent retinal thickening, or one disk area of retinal thickening any part of which is within one disk diameter of the center of the macula are defined as "Clinically relevant DME" ${ }^{52}$

\section{What is a Naïve Patient?}

In the strictest agreement with the term, the panel defined a naïve patient as

- A patient who, up to that moment, had never received any treatment (pharmacological, laser, and/or surgical).

However, sometimes, it might be considered a naïve patient when he/she has received non-macula involved laser photocoagulation. In other words, would be a naïve patient for either intravitreal therapy with anti-VEGF and/ or steroids or grid macular laser.

\section{Have Patients Treated with Anti-VEGF a Different Profile Than Those Treated with Sustained Released Corticosteroid Devices?}

The panel agreed that there are not different types of naïve patients, but rather different kinds of patients. Having said that, it is important to take into consideration that there are different profiles of naïve patients according to their disease evolution; metabolic control; retinal thickness, and/or visual acuity. Therefore, the therapeutic response will critically depend on the clinical and demographic patient characteristics. ${ }^{6-11}$

\section{What is a Refractory Patient?}

A DME refractory patient may be defined as the one who did not achieve a complete resolution of the disease, regardless of the treatment administered. ${ }^{52-56}$ 
Nevertheless, since anti-VEGF agents are broadly used as first-line therapy, the panel focused on the definition of DME patient's refractory to anti-VEGF or DME patients who do not adequately respond to anti-VEGF therapy.

- Patient refractory to anti-VEGF: A patient that after loading dose (three consecutive monthly injections), shows no improvement in visual acuity ( $>5$ ETDRS letters) and a $\leq 10 \%$ reduction of the central foveal thickness measured by optical coherence tomography $(\mathrm{OCT}){ }^{56}$ In a strict manner, the anatomical nonimprovement could be defined as a thickness reduction $<20 \%$ of CST.

Although we have defined a patient refractory to antiVEGF as a patient that after three consecutive monthly injections of anti-VEGF showed no functional and anatomic improvement, according to data from Protocol $\mathrm{T}$ it seemed that maintaining anti-VEGF treatment for 24 weeks might have positive outcomes on DME resolution. ${ }^{56}$ Therefore, extending the loading dose to five injections of intravitreal anti-VEGF, particularly with aflibercept, may be recommended.

\section{What is a Chronic DME Patient?}

To establish the definition of chronic DME is anything but easy.

We agreed to define chronic DME according to Bressler et $\mathrm{al}^{56}$ as

- Those eyes who did not achieve a CST $<250 \mu \mathrm{m}$ and/or $>10 \%$ reduction on at least two consecutive visits subsequent to the last follow-up visit.

Do the Different Patients' Profiles Require Different Therapeutic Goals?

The panel members fully agreed that, in order to obtain the best results, it is necessary to individualize the therapeutic objectives according to the patient profile.

\section{How Can We Define a Refractory ME? Does It Depend on the Patient Profile?}

According to the Diabetic Retinopathy Clinical Research Network (DRCR.net) ${ }^{56}$ and panel opinion, a refractory ME can be defined as:

- A VA improvement ( $\leq 5$ letters ETDRS) and a $\leq 10 \%$ reduction in CST (measured by OCT) . The anato- mical non-improvement could be also defined as a thickness reduction $<20 \%$ of CST.

Baseline patient profile may impact on treatment success: The better baseline characteristics (profile) the better treatment response. Better baseline conditions include good VA; younger age; absence of vitreous retinal alterations, like epiretinal membranes; status of the outer retina, and grade of diabetic retinopathy. ${ }^{57}$

\section{How Can We Define Lack of Treatment Response?}

According to the panel members, after administration of three anti-VEGF intravitreal injections, a non-response to an intravitreal treatment would be defined as:

- Persistence or worsening of DME.

- Non-improvement in functional or anatomic outcomes.

\section{Definition of Predictive and Prognostic Factor}

Although the terms "predictive" and "prognostic" factors have been commonly used in many studies, they are seldom defined and are often used interchangeably. ${ }^{58}$

About this subject, the definitions that reached a greater agreement among the panel members were:

- Prognostic factor: A characteristic that gives some information about the evolution of the patient. It can be helpful to guide the therapeutic approach. These factors are related to the evolution of the disease, and they are associated to the functional response that can be expected for the patient independently of the treatment administered.

- Predictive factor: A characteristic of the patient (clinical, diagnostic, genetic, etc.) that gives some information about the response (anatomical and/or functional) he/she will have to specific treatment. Predictive factors could be related to the probability of response to treatment.

\section{OCT Biomarkers}

Different OCT biomarkers, such as disorganization of the retinal inner layers (DRIL), hyperreflective dots (HRD), and epiretinal membranes may help clinicians to predict the effect of intravitreal therapy and may assist the choice of the pharmacological agent in the future. ${ }^{59}$ 
Which Parameters Should Be Evaluated at Baseline in a DME Patient?

OCT parameters that are important to analyze at baseline visit

- CMT.

- Cysts (number, localization, and size).

- HRD (number and localization).

- Inner and outer retinal layers.

- Outer nuclear layer.

- Serous retinal detachment (SRD).

- Vitreo-retinal interface.

\section{Serous Retinal Detachment (SRD)}

Based on OCT examinations, it is possible to define three different morphologic subtypes of DME, namely spongelike diffuse retinal thickening (DRT), cystoid macular edema (CME), and serous retinal detachment (SRD) ${ }^{60,61}$ (Figure 1).

It has been proposed an association between inflammation and the presence of SRD. ${ }^{62-64}$ Panel members agree with it. However, they consider that it is not clear whether the higher concentration of intravitreal cytokines is the cause or the consequence of SRD. Some papers propose a relationship between systemic diseases, such as chronic kidney disease, or glycemic control and SRD. ${ }^{65,66}$ Panel members do not consider SRD as a prognostic factor itself. However, long-term edemas usually present higher concentration of inflammatory cytokines and SRD is more frequent in this type of edemas. Therefore, SRD might be correlated with chronicity and worse type of edema.

Regarding the question of whether SRD is a predictive factor of response to intravitreal treatment, currently available scientific evidence provides conflicting results. While some studies have reported that the presence of SRD was associated with better functional and/or anatomic results to anti-VEGF treatments, ${ }^{39,67,68}$ others have reported no better responsiveness with the same treatments. ${ }^{69-71}$

In addition, there is evidence suggesting that dexamethasone intravitreal (DEX) implants might provide better functional and/or anatomic outcomes in eyes with SRD. ${ }^{38,40,72}$ In this sense, panel members recommend to use DEX implants rather than anti-VEGF for treating DME with SRD, especially the chronic ones. However, other factors, such as lens status, responsiveness to steroids (elevation of intraocular pressure), and glaucoma should be taken into consideration.

\section{Cystoid Macular Edema}

Cyst formation begins with intercellular fluid accumulation, although it is not clear if the cystoid spaces are intracellular or extracellular to the Müller cells. ${ }^{60,61,73,74}$

According to evidence and panel opinion, the presence of cysts is a marker of disease activity, chronicity of DME, and/or structural damage. ${ }^{56,75,76}$

Panel members agreed that, up to now, there is not enough scientific evidence to support the use of a specific treatment based on the number, size, or location of the cysts.

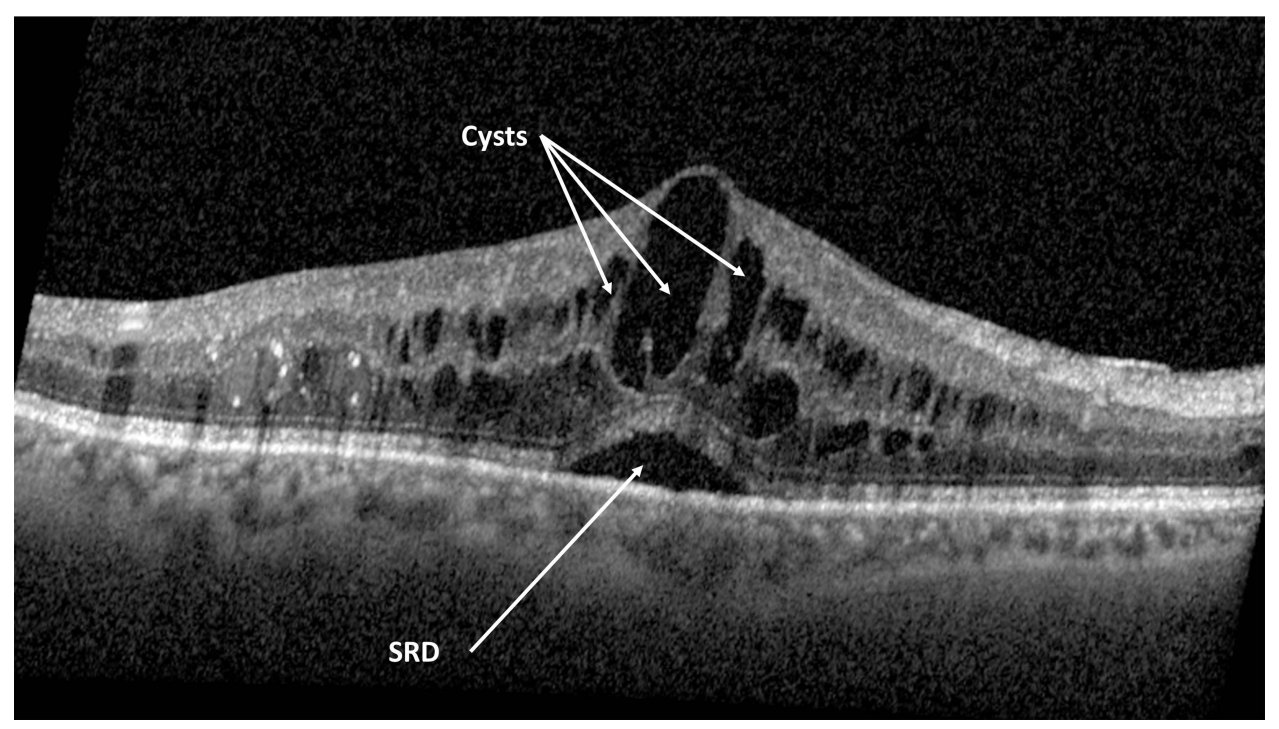

Figure I Spectral domain optical coherence tomography image of an eye with cystoid macular edema (CME) and serous retinal detachment (SRD). 
Additionally, they all agreed that large empty cysts are usually related to more advanced stages of the disease and may be a sign of chronicity. Therefore, DEX implants might be an option as first-line therapy.

\section{Hyperreflective Dots (HRD)}

The presence of HRD as image biomarkers has been suggested in DME. ${ }^{77}$ The role of the HRDs in predicting clinical outcomes in patients undergoing treatment for macular edema has shown controversial results. While some studies have shown that the presence of HRDs was associated with poorer visual outcome in patients with macular edema, ${ }^{78,79}$ one study reported that a higher number of HRDs at baseline was associated with an adequate treatment response. ${ }^{80}$

Although the etiology of HRDs has not been fully elucidated, retinal inflammation seemed to be involved. ${ }^{81}$ Panel members agreed with it and based on this assumption, DEX implant would be the treatment of choice.

However, they seem to be a marker of bad prognosis; therefore, the functional response might be limited.

\section{Disorganization of the Retinal Inner Layers (DRIL) and Outer Retinal Layer (ORL) \\ DRIL}

Disorganization of the retinal inner layers (DRIL) has been identified as a prognostic factor in DME patients. ${ }^{82,83}$ In fact, there seems to be an association between DRIL resolution and best corrected VA (BCVA) improvement. $^{82-84}$

Although the panel strongly recommended to assess the presence of DRIL at baseline, they are aware that it is not always easy to evaluate, that is why it is recommended to evaluate DRIL at follow up visits (Figure 2A).

Additionally, DRIL used to be associated with damage on other retinal layers like ellipsoid zone (EZ) and external limiting membrane (ELM). ${ }^{83}$ Since the presence of DRIL indicates chronicity, panel members agree that treatment should be switched early if patient do not respond properly.

Recent scientific evidence has suggested that DEX implant may effectively ameliorate DRIL. ${ }^{85}$

Outer retinal layer (ORL)

ORL is the distance between ELM and retinal pigmented epithelium (RPE), which is the length of both inner and outer segment of the photoreceptor layer (Figure 2B and $\mathrm{C}$ ). The main reason for delayed or incomplete visual recovery seems to be related to ultrastructural changes of the outer retinal layers. ${ }^{86-89}$ Although DME may be treated effectively in many eyes, outer retinal structures may remain irreversibly damaged in some patients. ${ }^{88}$

ELM integrity was associated with a final BCVA improvement in DME patients. ${ }^{88,89}$ This may suggest a significant relationship between ELM integrity and photoreceptor cell bodies' status, which may be a sign of advanced photoreceptor damage. ${ }^{88,89}$ Moreover, ORL thickness correlates better with vision than the total retinal thickness. ${ }^{90-92}$

Additionally, in eyes with DME refractory to antiVEGF therapy who received treatment with DEX implant, ORL disruptions might predict smaller VA gains if evaluated after an initial reduction of DME that DEX implant may effectively recover morphology of ORL in DME patients. ${ }^{93,94}$

Intercellular adhesion molecule-1 (ICAM-1) is an immunoglobulin that has been implicated in the development of leukostasis, a relevant feature of DR. ${ }^{95}$ In fact, it has been suggested that both increased levels of VEGF and ICAM-1 are involved in DR development and are responsible of the ELM and of the inner segment and outer segment (IS/OS) junction disruption. ${ }^{95}$

Moreover, in patients with DME, intravitreal bevacizumab has been associated with a restoration of the ELM, which was followed by a restoration of the EZ. ${ }^{96}$

In patients with uveitic cystoid macular edema, DEX implant was able to reverse ORL alterations. ${ }^{97}$ These findings were also observed in patients with macular edema associated with branch retinal vein occlusion. ${ }^{98}$

There is an increasing evidence suggesting that DEX implant may effectively recover the morphology of ORL in DME patients, which might be associated with better functional outcomes. ${ }^{93,94,99,100}$

Panel recommendation:

- ORL disruptions have a stronger prognostic value than DRIL in eyes with DME.

- Since ORL plays an important role as prognostic factor, it needs to be assessed at baseline. Despite the advances in OCT technology, in patients with "big DME", it could be difficult to properly evaluate all the layers, so it would be necessary to reassess at follow-up visit. If ORL does not improve at all after one DEX implant, panel members agree that functional prognosis is very poor.

- Despite the promising results obtained with DEX implants, more evidence would be needed determining the best treatment option in DME eyes with 

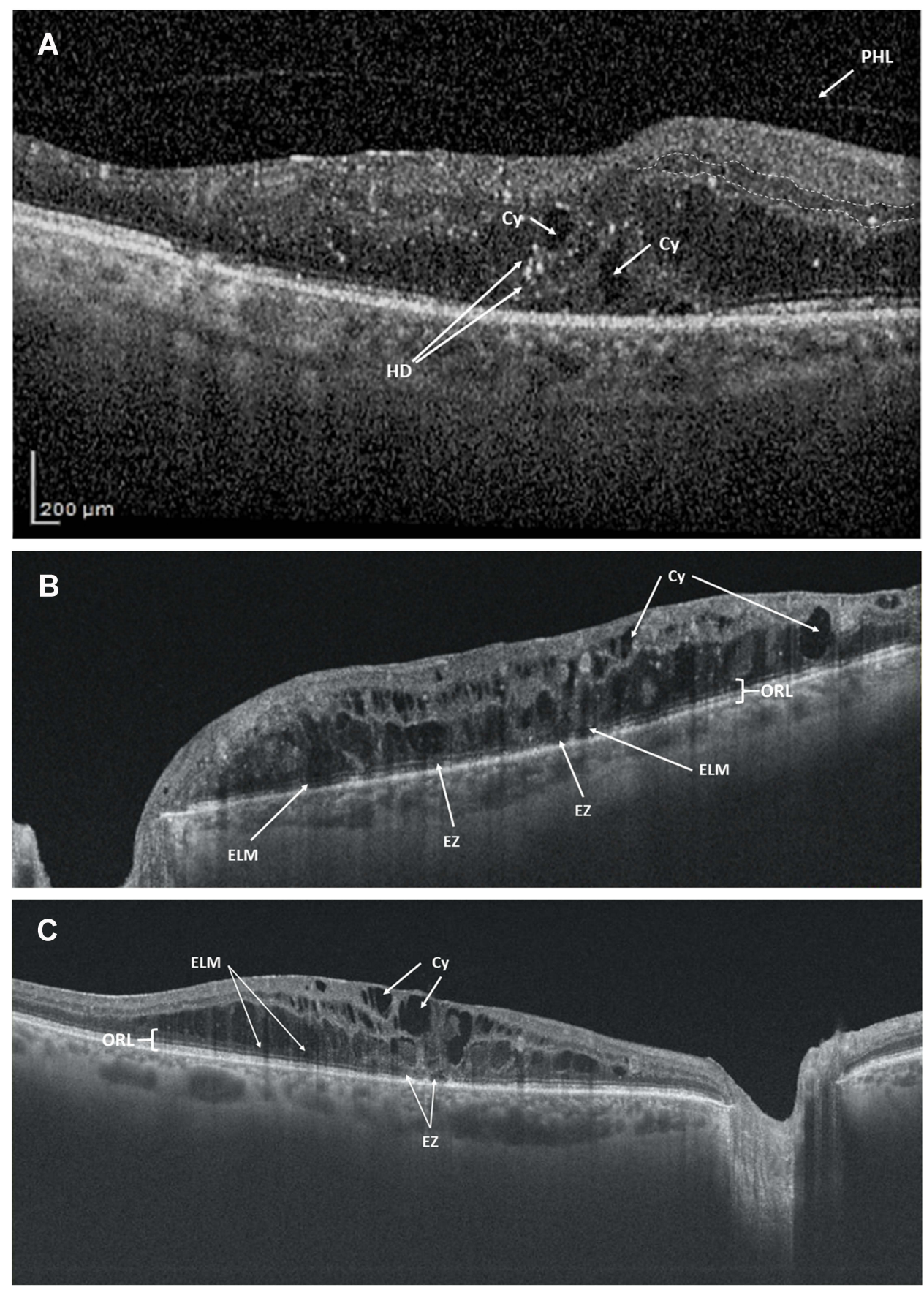

Figure 2 Spectral domain optical coherence tomography images. (A) Disorganization of the retinal inner layers (DRIL). It is not easy to identify the boundaries of the inner layers (white dotted line). Additionally, it is possible to see cysts (Cy) and hyperreflective dots (HD) in the outer layers, as well as the posterior hyaloid (PHL). (B) Spongiform edema with damage in the outer (ORL) and inner (INL) retinal layers. Besides the presence of cysts (Cy), it is possible to identify the external limiting membrane (ELM), and ellipsoid zone (EZ) disruptions. (C) Cystoid macular edema with external limiting membrane disruptions (ELMD). Additionally, it is possible to see some damage in the ellipsoid zone (EZ) and some cysts (Cy).

Abbreviation: ORL, outer retinal layers.

DRIL/ORL. Nevertheless, it should be taken into consideration that DEX implant provides a rapid anatomic response, which might facilitate an adequate assessment of ORL status.

\section{Outer Nuclear Layer}

The outer nuclear layer (ONL) contains the rod and cone cell bodies. ONL thickness is increased in diabetic patients. ${ }^{101,102}$ ONL damage is a marker of bad visual 
prognosis in DME patients and therefore the functional response is usually limited. ${ }^{89,103,104}$

It has been suggested that DEX implant may recover morphology of ONL in DME patients, which was associated with better functional outcomes. ${ }^{104}$ However, further research is needed to confirm this finding.

Panel recommendation:

- Since functional response is usually limited in DME with ONL damage, intravitreal anti-VEGF injections or DEX implant could be indistinctly used as first-choice therapy. ONL alterations may be considered as a sign of bad prognosis. There is evidence suggesting that DEX implant might recover ONL damage, which, therefore, was associated with better functional outcomes. ${ }^{104}$ However, further research is needed to confirm this finding.

\section{Central Macular Thickness}

Although CMT reduction, measured with OCT, is a very useful marker and is commonly used for monitoring treatment response in eyes with DME, there is not enough scientific evidence to support the relationship between CMT and chronicity or CMT and inflammation in DME. ${ }^{6-15,40,41}$

Panel recommendation:

- CMT has to be assessed at baseline. DEX implant may be a first choice in eyes with diffuse DME, since they may be associated with a greater inflammatory component. ${ }^{105}$ Additionally, there is evidence suggesting a significant association between macular thickness and the concentration of inflammatory marker ICAM-1. ${ }^{106}$ However, other factors need to be considered to decide the best treatment option.

Table 1 summarizes the main findings and comments of the panel regarding biomarkers.

Table I Overview of the Role of the Different Biomarkers Comments are based on the expert panel members experience as well as currently available scientific evidence [see references ${ }^{60-106}$ ]

\begin{tabular}{|c|c|c|c|c|c|}
\hline & Inflammatory Marker & Prognosis* & $\begin{array}{l}\text { Chronicity } \\
\text { Marker }\end{array}$ & $\begin{array}{l}\text { Predictor of } \\
\text { Response to } \\
\text { DEX }\end{array}$ & $\begin{array}{l}\text { Candidate for } \\
\text { First-Line } \\
\text { Treatment with } \\
\text { DEX }\end{array}$ \\
\hline SRD & Yes & $\begin{array}{l}\text { Good/bad prognosis (depends on time } \\
\text { course and other biomarkers) }\end{array}$ & No & Yes & Yes \\
\hline CYSTS & $\begin{array}{l}\text { No (cysts with Hyperreflective } \\
\text { material inside, have a greater } \\
\text { inflammatory component) }\end{array}$ & $\begin{array}{l}\text { Good/bad prognosis (depends on number, } \\
\text { size, location and chronicity. Dense content } \\
\text { is a sign of better prognosis) }\end{array}$ & $\begin{array}{l}\text { Yes (if big } \\
\text { cysts are } \\
\text { present) }\end{array}$ & $\begin{array}{c}\text { Yes } \\
\text { (intraretinal } \\
\text { inflammatory } \\
\text { cysts) }\end{array}$ & Yes \\
\hline HRD & Yes & Bad prognosis & $\begin{array}{l}\text { Yes (in } \\
\text { general } \\
\text { terms) }\end{array}$ & Yes & Yes \\
\hline DRIL & No & Bad prognosis & Yes & No & No \\
\hline ORL & No & Bad prognosis & Yes & No & No \\
\hline ONL & No & Bad prognosis & Yes & No & $\begin{array}{l}\text { No (DEX and anti- } \\
\text { VEFG } \\
\text { indistinctively) }\end{array}$ \\
\hline $\mathrm{CMT}^{+}$ & No & Bad prognosis & No & $\begin{array}{l}\text { No (an } \\
\text { exception in } \\
\text { patients with } \\
\text { SRD) }\end{array}$ & $\begin{array}{l}\text { Yes (in cases with } \\
\text { high TMV) }\end{array}$ \\
\hline
\end{tabular}

Notes: *Its presence is a sign of ... + big volume.

Abbreviations: DEX, dexamethasone intravitreal implant; SRD, serous retinal detachment; HRD, hyperreflective dots; DRIL, disorganization of the retinal inner layers; ORL, outer retinal layer; ONL, outer nuclear layer; CMT, central macular thickness; anti-VEGF, vascular endothelial growth factor inhibitors; TMV, total macular volume. 


\section{Conclusions}

As therapeutic objectives, the panel recommended in patients with good baseline VA to maintain it and improve it if possible, taking into account the ceiling effect, and in patients with low baseline VA to improve it as much as possible except in patients with severe retinal damage that could limit the functional improvement; in those cases, maintenance of vision should be the main objective. From a daily practice perspective, the panel recommended, as main therapeutic objective to achieve the best anatomic response as fast as possible, and at that point we have to check the maximum functional response the patient can get.

According to the panel, DME treatment should start at soon as possible, according to the Retina Unit capability.

According to the panel, after administration of three anti-VEGF intravitreal injections, no response to an intravitreal treatment would be defined as:

Persistence or worsening of DME and/or nonimprovement in functional or anatomic outcomes.

Different biomarkers, including SRD, DRIL, cysts, HRD, ORL and ONL alterations, and CMT have been identified as prognostic and predictive factors in patients with DME. The panel recommended to assess these biomarkers and take them into consideration when selecting the therapeutic strategy.

As a limitation of the current consensus, it should be mentioned the lack of discussion about costs-effectiveness of the different treatment options or social impact of both disease impairment and treatment. It is well known that both DME and diabetic retinopathy have a significant economic impact due to their direct and indirect costs, including reduction in income or an increased need for social support as vision worsens. ${ }^{107}$ Data about economic burden of DME in Spain suggested that the estimated direct annual cost per patient with DME was $€ 6271$ (excluding drug costs), ${ }^{108}$ while the estimated annual cost of treatment with anti-VEGF was $€ 7154 .{ }^{106,109}$

This consensus has highlighted different aspects related to the management of DME patients in daily clinical practice.

\section{Acknowledgments}

Medical writing and editorial assistance services have been provided by Antonio Martínez (MD) of Ciencia y Deporte S.L. and covered by a Grant from Allergan. Support for this assistance was funded by Allergan, an AbbVie company.

\section{Author Contributions}

All authors contributed to data analysis, drafting or revising the article, gave final approval of the version to be published, agreed to the submitted journal, and agreed to be accountable for all aspects of the work.

\section{Funding}

Logistic for writing services has been provided by Allergan, an AbbVie company. Allergan did not participate in either data analysis or redaction of the manuscript.

\section{Disclosure}

Dr Patricia Udaondo reports grants from Allergan, an AbbVie company, during the conduct of the study. Dr Veronica CastroNavarro reports grants from Allergan, an AbbVie company, over the last year. Dr José María Ruiz Moreno reports grants from Allergan, an AbbVie company, over the last year. Dr Javier Ascaso reports personal fees from Allergan, personal fees from Novartis, personal fees from Bayer, personal fees from Brill Pharma, personal fees from Alcon, outside the submitted work. Dr Alfredo García-Layana reports personal fees from Allergan, personal fees from Novartis, personal fees from Bayer, personal fees from Roche, grants, personal fees from Thea, during the conduct of the study. Dr Francisco Javier Lavid reports personal fees from Allergan, during the conduct of the study; personal fees from Novartis, personal fees from Bayer, personal fees from Allergan, outside the submitted work. The authors report no other conflicts of interest in this work.

\section{References}

1. Cho NH, Shaw JE, Karuranga S, et al. IDF Diabetes Atlas: global estimates of diabetes prevalence for 2017 and projections for 2045 . Diabetes Res Clin Pract. 2018;138:271-281. doi:10.1016/j. diabres.2018.02.023

2. Bourne RRA, Jonas JB, Bron AM, et al.; Vision Loss Expert Group of the Global Burden of Disease Study. Prevalence and causes of vision loss in high-income countries and in Eastern and Central Europe in 2015: magnitude, temporal trends and projections. Br J Ophthalmol. 2018;102(5):575-585. doi:10.1136/bjophthalmol-2017-311258.

3. Li JQ, Welchowski T, Schmid M, et al. Prevalence, incidence and future projection of diabetic eye disease in Europe: a systematic review and meta-analysis. Eur $J$ Epidemiol. 2020;35(1):11-23. doi:10.1007/s10654-019-00560-Z

4. Kocur I, Resnikoff S. Visual impairment and blindness in Europe and their prevention. Br J Ophthalmol. 2002;86(7):716-722. doi:10.1136/ bjo.86.7.716

5. Cunha-Vaz J, Coscas G. Diagnosis of macular edema. Ophthalmologica. 2010;224(Suppl 1):2-7. doi:10.1159/000315156

6. Barham R, El Rami H, Sun JK, Silva PS. Evidence-based treatment of diabetic macular edema. Semin Ophthalmol. 2017;32(1):56-66. doi:10.1080/08820538.2016.1228388 
7. Schmidt-Erfurth U, Garcia-Arumi J, Bandello F, et al. Guidelines for the management of diabetic macular edema by the European Society of Retina Specialists (EURETINA). Ophthalmologica. 2017;237(4):185-222. doi:10.1159/000458539

8. García-Layana A, Figueroa MS, Arias L, et al. Clinical decision-making when treating diabetic macular edema patients with dexamethasone intravitreal implants. Ophthalmologica. 2018;240(2):61-72. doi:10.1159/000486800

9. Kim EJ, Lin WV, Rodriguez SM, Chen A, Loya A, Weng CY. Treatment of diabetic macular edema. Curr Diab Rep. 2019;19 (9):68. doi:10.1007/s11892-019-1188-4

10. Kodjikian L, Bellocq D, Bandello F, et al. First-line treatment algorithm and guidelines in center-involving diabetic macular edema. Eur J Ophthalmol. 2019;29(6):573-584. doi:10.1177/ 1120672119857511

11. Singh RP, Elman MJ, Singh SK, Fung AE, Stoilov I. Advances in the treatment of diabetic retinopathy. $J$ Diabetes Complications. 2019;33(12):107417. doi:10.1016/j.jdiacomp.2019.107417

12. Acón D, Wu L. Multimodal Imaging in Diabetic Macular Edema. Asia Pac J Ophthalmol. 2018;7(1):22-27. doi:10.22608/ APO.2017504

13. Liu G, Xu D, Wang F. New insights into diabetic retinopathy by OCT angiography. Diabetes Res Clin Pract. 2018;142:243-253. doi:10.1016/j.diabres.2018.05.043

14. Wong TY, Sun J, Kawasaki R, et al. Guidelines on Diabetic Eye Care: the International Council of Ophthalmology Recommendations for Screening, Follow-up, Referral, and Treatment Based on Resource Settings. Ophthalmology. 2018;125(10):1608-1622. doi:10.1016/j.ophtha.2018.04.007

15. Kwan CC, Fawzi AA. Imaging and biomarkers in diabetic macular edema and diabetic retinopathy. Curr Diab Rep. 2019;19 (10):95. doi:10.1007/s11892-019-1226-2

16. Early Treatment Diabetic Retinopathy Study Research Group: Treatment techniques and clinical guidelines for photocoagulation of diabetic macular edema. Early Treatment Diabetic Retinopathy Study Report Number 2. Ophthalmology. 1987;94:761-774. doi:10.1016/s0161-6420(87)33527-4

17. Early Treatment Diabetic Retinopathy Study Research Group: Focal photocoagulation treatment of diabetic macular edema. Relationship of treatment effect to fluorescein angiographic and other retinal characteristics at baseline: ETDRS report No. 19. Arch Ophthalmol. 1995;113:1144-1155. doi:10.1001/ archopht. 1995.01100090070025

18. Cunningham ET, Adamis AP, Altaweel M, et al.; Macugen Diabetic Retinopathy Study Group. A Phase II randomized double-masked trial of pegaptanib, an anti-vascular endothelial growth factor aptamer, for diabetic macular edema. Ophthalmology. 2005;112(10):1747-1757.

19. Sultan MB, Zhou D, Loftus J, Dombi T, Ice KS; Macugen 1013 Study Group. A Phase 2/3, multicenter, randomized, double-masked, 2-year trial of pegaptanib sodium for the treatment of diabetic macular edema. Ophthalmology. 2011;118 (6):1107-1118. doi:10.1016/j.ophtha.2011.02.045

20. Wells JA, Glassman AR, Ayala AR, et al.; Diabetic Retinopathy Clinical Research Network. Aflibercept, bevacizumab, or ranibizumab for diabetic macular edema. $N$ Engl J Med. 2015;372 (13):1193-1203.

21. Wells JA, Glassman AR, Ayala AR, et al.; Diabetic Retinopathy Clinical Research Network. Aflibercept, bevacizumab, or ranibizumab for diabetic macular edema: two-year results from a comparative effectiveness randomized clinical trial. Ophthalmology. 2016;123(6):1351-1359. doi:10.1016/j. ophtha.2016.02.022.
22. Nguyen QD, Brown DM, Marcus DM, et al.; RISE and RIDE Research Group. Ranibizumab for diabetic macular edema: results from 2 Phase III randomized trials: RISE and RIDE. Ophthalmology. 2012;119(4):789-801. doi:10.1016/j.ophtha.2011.12.039.

23. Brown DM, Nguyen QD, Marcus DM, et al.; RIDE and RISE Research Group. Long-term outcomes of ranibizumab therapy for diabetic macular edema: the 36-month results from two phase III trials: RISE and RIDE. Ophthalmology. 2013;120 (10):2013-2022. doi:10.1016/j.ophtha.2013.02.034.

24. Boyer DS, Nguyen QD, Brown DM, Basu K, Ehrlich JS; RIDE and RISE Research Group. Outcomes with as-needed ranibizumab after initial monthly therapy: long-term outcomes of the phase III RIDE and RISE Trials. Ophthalmology. 2015;122 (12):2504-2513.e1. doi:10.1016/j.ophtha.2015.08.006

25. Korobelnik JF, Do DV, Schmidt-Erfurth U, et al. Intravitreal aflibercept for diabetic macular edema. Ophthalmology. 2014;121(11):2247-2254. doi:10.1016/j.ophtha.2014.05.006

26. Brown DM, Schmidt-Erfurth U, Do DV, et al. Intravitreal aflibercept for diabetic macular edema: 100- week results from the VISTA and VIVID studies. Ophthalmology. 2015;122:2044-2052. doi:10.1016/j.ophtha.2015.06.017

27. Do DV, Nguyen QD, Vitti R, et al. Intravitreal aflibercept injection in diabetic macular edema patients with and without prior anti-vascular endothelial growth factor treatment: outcomes from the phase 3 program. Ophthalmology. 2016;123(4):850-857. doi:10.1016/j.ophtha.2015.11.008

28. Boyer DS, Faber D, Gupta S, et al.; Ozurdex CHAMPLAIN Study Group. Dexamethasone intravitreal implant for treatment of diabetic macular edema in vitrectomized patients. Retina. 2011;31(5):915-923. doi:10.1097/IAE.0b013e318206d18c.

29. Callanan DG, Gupta S, Boyer DS, et al.; Ozurdex PLACID Study Group. Dexamethasone intravitreal implant in combination with laser photocoagulation for the treatment of diffuse diabetic macular edema. Ophthalmology. 2013;120(9):1843-1851. doi:10.1016/ j.ophtha.2013.02.018.

30. Gillies MC, Lim LL, Campain A, et al. A randomized clinical trial of intravitreal bevacizumab versus intravitreal dexamethasone for diabetic macular edema: the BEVORDEX study. Ophthalmology. 2014;121(12):2473-2481. doi:10.1016/j.ophtha.2014.07.002

31. Fraser-Bell S, Lim LL, Campain A, et al. Bevacizumab or Dexamethasone Implants for DME: 2-year Results (The BEVORDEX Study). Ophthalmology. 2016;123(6):1399-1401. doi:10.1016/j.ophtha.2015.12.012

32. Aroney C, Fraser-Bell S, Lamoureux EL, Gillies MC, Lim LL, Fenwick EK. Vision-Related quality of life outcomes in the bevordex study: a clinical trial comparing ozurdex sustained release dexamethasone intravitreal implant and bevacizumab treatment for diabetic macular edema. Invest Ophthalmol Vis Sci. 2016;57(13):5541-5546. doi:10.1167/iovs.16-19729

33. Campochiaro PA, Brown DM, Pearson A, et al.; FAME Study Group. Long-term benefit of sustained-delivery fluocinolone acetonide vitreous inserts for diabetic macular edema. Ophthalmology. 2011;118(4):626-635.e2. doi:10.1016/j.ophtha.2010.12.028.

34. Cunha-Vaz J, Ashton P, Iezzi R, et al.; FAME Study Group. Sustained delivery fluocinolone acetonide vitreous implants: long-term benefit in patients with chronic diabetic macular edema. Ophthalmology. 2014;121(10):1892-1903. doi:10.1016/j. ophtha.2014.04.019.

35. Baker CW, Glassman AR, Beaulieu WT, et al.; DRCRRetina Network. Effect of initial management with aflibercept vs laser photocoagulation vs observation on vision loss among patients with diabetic macular edema involving the center of the macula and good visual acuity: a randomized clinical trial. JAMA. 2019;321(19):1880-1894. doi:10.1001/jama.2019.5790. 
36. Reznicek L, Cserhati S, Seidensticker F, et al. Functional and morphological changes in diabetic macular edema over the course of anti-vascular endothelial growth factor treatment. Acta Ophthalmol. 2013;91(7):e529-536. doi:10.1111/aos.12153

37. Matsuda S, Tam T, Singh RP, et al. The impact of metabolic parameters on clinical response to VEGF inhibitors for diabetic macular edema. J Diabetes Complications. 2014;28(2):166-170. doi:10.1016/j.jdiacomp.2013.11.009

38. Zur D, Iglicki $M$, Busch $C$, Invernizzi A, Mariussi $M$, Loewenstein A; International Retina Group. OCT biomarkers as functional outcome predictors in diabetic macular edema treated with dexamethasone implant. Ophthalmology. 2018;125 (2):267-275. doi:10.1016/j.ophtha.2017.08.031

39. Gerendas BS, Prager S, Deak G, et al. Predictive imaging biomarkers relevant for functional and anatomical outcomes during ranibizumab therapy of diabetic macular oedema. $\mathrm{Br}$ $J$ Ophthalmol. 2018;102(2):195-203. doi:10.1136/bjophthalmol2017-310483

40. Lee H, Kang KE, Chung H, Kim HC. Prognostic factors for functional and anatomic outcomes in patients with diabetic macular edema treated with dexamethasone implant. Korean J Ophthalmol. 2018;32(2):116-125. doi:10.3341/kjo.2017.0041

41. Lee H, Kang KE, Chung H, Kim HC. Three-dimensional analysis of morphologic changes and visual outcomes in diabetic macular edema. Jpn J Ophthalmol. 2019;63(3):234-242. doi:10.1007/ s10384-019-00657-8

42. Do DV, Cho M, Nguyen QD, et al. Impact of optical coherence tomography on surgical decision making for epiretinal membranes and vitreomacular traction. Retina. 2007;27(5):552-556 doi:10.1097/IAE.0b013e31802c518b

43. Sakamoto A, Nishijima K, Kita M, Oh H, Tsujikawa A, Yoshimura N. Association between foveal photoreceptor status and visual acuity after resolution of diabetic macular edema by pars plana vitrectomy. Graefes Arch Clin Exp Ophthalmol. 2009;247(10):1325-1330. doi:10.1007/s00417-009-1107-5

44. Alasil T, Keane PA, Updike JF, et al. Relationship between optical coherence tomography retinal parameters and visual acuity in diabetic macular edema. Ophthalmology. 2010;117 (12):2379-2386. doi:10.1016/j.ophtha.2010.03.051

45. Maheshwary AS, Oster SF, Yuson RM, Cheng L, Mojana F, Freeman WR. The association between percent disruption of the photoreceptor inner segment-outer segment junction and visual acuity in diabetic macular edema. Am J Ophthalmol. 2010;150 (1):63-67.e1. doi:10.1016/j.ajo.2010.01.039

46. Dugel PU, Campbell JH, Kiss S, et al. Association between early anatomic response to anti-vascular endothelial growth factor therapy and long-term outcome in diabetic macular edema: an independent analysis of protocol I study data. Retina. 2019;39 (1):88-97. doi:10.1097/IAE.0000000000002110

47. Busch C, Fraser-Bell S, Zur D, et al.; International Retina Group. Real-world outcomes of observation and treatment in diabetic macular edema with very good visual acuity: the OBTAIN study. Acta Diabetol. 2019;56(7):777-784. doi:10.1007/s00592019-01310-z.

48. Wykoff CC. Thresholds for initiating treatment of eyes with diabetic macular edema and good vision: consideration of DRCR.Net Protocol V Results. Ophthalmol Retina. 2019;3 (11):917-919. doi:10.1016/j.oret.2019.07.007

49. Wells JA, Glassman AR, Jampol LM, et al.; Diabetic Retinopathy Clinical Research Network. Association of baseline visual acuity and retinal thickness with 1-year efficacy of aflibercept, bevacizumab, and ranibizumab for diabetic macular edema. JAMA Ophthalmol. 2016;134(2):127-134. doi:10.1001/ jamaophthalmol.2015.4599.
50. Cantrell RA, Lum F, Chia Y, et al. Treatment patterns for diabetic macular edema: an intelligent research in sight (IRIS ${ }^{\circledR}$ ) registry analysis. Ophthalmology. 2020;127(3):427-429. doi:10.1016/j. ophtha.2019.10.019

51. Sadda SR, Campbell J, Dugel PU, et al. Relationship between duration and extent of oedema and visual acuity outcome with ranibizumab in diabetic macular oedema: a post hoc analysis of Protocol I data. Eye. 2020;34(3):480-490. doi:10.1038/s41433019-0522-z

52. Browning DJ, Stewart MW, Lee C. Diabetic macular edema: evidence-based management. Indian J Ophthalmol. 2018;66 (12):1736-1750. doi:10.4103/ijo.IJO_1240_18

53. Rosenblatt BJ, Shah GK, Sharma S, Bakal J. Pars plana vitrectomy with internal limiting membranectomy for refractory diabetic macular edema without a taut posterior hyaloid. Graefes Arch Clin Exp Ophthalmol. 2005;243(1):20-25. doi:10.1007/s00417004-0958-z

54. Patel JI, Hykin PG, Schadt M, Luong V, Fitzke F, Gregor ZJ. Pars plana vitrectomy for diabetic macular oedema: OCT and functional correlations. Eye. 2006;20(6):674-680. doi:10.1038/sj. eye. 6701945

55. Maturi RK, Glassman AR, Liu D, et al.; Diabetic Retinopathy Clinical Research Network. Effect of Adding dexamethasone to continued ranibizumab treatment in patients with persistent diabetic macular edema: a drcr network phase 2 randomized clinical trial. JAMA Ophthalmol. 2018;136(1):29-38. doi:10.1001/ jamaophthalmol.2017.4914.

56. Bressler NM, Beaulieu WT, Glassman AR, et al.; Diabetic Retinopathy Clinical Research Network. Persistent macular thickening following intravitreous aflibercept, bevacizumab, or ranibizumab for central-involved diabetic macular edema with vision impairment: a secondary analysis of a randomized clinical trial. JAMA Ophthalmol. 2018;136(3):257-269. doi:10.1001/ jamaophthalmol.2017.6565.

57. Chen YP, Wu AL, Chuang CC, Chen SN. Factors influencing clinical outcomes in patients with diabetic macular edema treated with intravitreal ranibizumab: comparison between responder and non-responder cases. Sci Rep. 2019;9(1):10952. doi:10.1038/ s41598-019-47241-1

58. National cancer institute dictionary of cancer terms. Available from: https://www.cancer.gov/publications/dictionaries/cancerterms/def/prognostic-factor. Accessed May 13, 2021.

59. Haritoglou C, Maier M, Neubauer AS, Augustin AJ. Current concepts of pharmacotherapy of diabetic macular edema. Expert Opin Pharmacother. 2020;21(4):467-475. doi:10.1080/ 14656566.2020.1713093

60. Otani T, Kishi S, Maruyama Y. Patterns of diabetic macular edema with optical coherence tomography. Am J Ophthalmol. 1999;127(6):688-693. doi:10.1016/S0002-9394(99)00033-1

61. Kim BY, Smith SD, Kaiser PK. Optical coherence tomographic patterns of diabetic macular edema. Am J Ophthalmol. 2006;142 (3):405-412. doi:10.1016/j.ajo.2006.04.023

62. Sonoda S, Sakamoto T, Yamashita T, Shirasawa M, Otsuka H, Sonoda Y. Retinal morphologic changes and concentrations of cytokines in eyes with diabetic macular edema. Retina. 2014;34 (4):741-748. doi:10.1097/IAE.0b013e3182a48917

63. Bandyopadhyay S, Bandyopadhyay SK, Saha M, Sinha A. Study of aqueous cytokines in patients with different patterns of diabetic macular edema based on optical coherence tomography. Int Ophthalmol. 2018;38(1):241-249. doi:10.1007/s10792-0170453-2

64. Yenihayat F, Özkan B, Kasap M, et al. Vitreous IL-8 and VEGF levels in diabetic macular edema with or without subretinal fluid. Int Ophthalmol. 2019;39(4):821-828. doi:10.1007/s10792-0180874-6 
65. Tsai MJ, Hsieh YT, Shen EP, Peng YJ. Systemic associations with residual subretinal fluid after ranibizumab in diabetic macular edema. J Ophthalmol. 2017;2017:4834201. doi:10.1155/2017/ 4834201

66. Turgut B, Gul FC, Ilhan N, Demir T, Celiker U. Comparison of serum glycosylated hemoglobin levels in patients with diabetic cystoid macular edema with and without serous macular detachment. Indian J Ophthalmol. 2010;58(5):381-384.

67. Sophie R, Lu N, Campochiaro PA. Predictors of functional and anatomic outcomes in patients with diabetic macular edema treated with ranibizumab. Ophthalmology. 2015;122(7):1395-1401. doi:10.1016/j.ophtha.2015.02.036

68. Fickweiler W, Schauwvlieghe AME, Schlingemann RO; BRDME Research Group. Predictive value of optical coherence tomographic features in the Bevacizumab and Ranibizumab in patients with Diabetic Macular Edema (BRDME) sTUDY. Retina. 2018;38(4):812-819. doi:10.1097/IAE.0000000000001626

69. Shimura M, Yasuda K, Yasuda M, Nakazawa T. Visual outcome after intravitreal bevacizumab depends on the optical coherence tomographic patterns of patients with diffuse diabetic macular edema. Retina. 2013;33(4):740-747. doi:10.1097/ IAE.0b013e31826b6763

70. Kaya M, Karahan E, Ozturk T, Kocak N, Kaynak S. Effectiveness of intravitreal ranibizumab for diabetic macular edema with serous retinal detachment. Korean J Ophthalmol. 2018;32 (4):296-302. doi:10.3341/kjo.2017.0117

71. Hwang HB, Jee D, Kwon JW. Characteristics of diabetic macular edema patients with serous retinal detachment. Medicine. 2019;98 (51):e18333. doi:10.1097/MD.0000000000018333

72. Castro-Navarro V, Cervera-Taulet E, Navarro-Palop C, Monferrer-Adsuara C, Hernández-Bel L, Montero-Hernández J. Intravitreal dexamethasone implant Ozurdex ${ }^{\circledR}$ in naïve and refractory patients with different subtypes of diabetic macular edema. BMC Ophthalmol. 2019;19(1):15.

73. Spaide RF. Retinal vascular cystoid macular edema: review and new theory. Retina. 2016;36(10):1823-1842. doi:10.1097/ IAE.0000000000001158

74. Sacconi R, Lutty GA, Mullins RF, Borrelli E, Bandello F, Querques G. Subretinal pseudocysts: a novel OCT finding in diabetic macular edema. Am J Ophthalmol Case Rep. 2019;16:100567. doi:10.1016/j.ajoc.2019.100567

75. Yamamoto S, Yamamoto $\mathrm{T}$, Hayashi $\mathrm{M}$, Takeuchi $\mathrm{S}$. Morphological and functional analyses of diabetic macular edema by optical coherence tomography and multifocal electroretinograms. Graefes Arch Clin Exp Ophthalmol. 2001;239(2):96-101. doi:10.1007/s004170000238

76. Bezzina AD, Carbonaro F. Factors Predicting treatment response in anti-vascular endothelial growth factor naïve diabetic macular edema patients treated with intravitreal bevacizumab. J Ocul Pharmacol Ther. 2019;35(10):551-557. doi:10.1089/ jop. 2018.0112

77. De Benedetto U, Sacconi R, Pierro L, Lattanzio R, Bandello F. Optical coherence tomographic hyperreflective foci in early stages of diabetic retinopathy. Retina. 2015;35(3):449-453. doi:10.1097/IAE.0000000000000336

78. Chatziralli IP, Sergentanis TN, Sivaprasad S. Hyperreflective foci as an independent visual outcome predictor in macular edema due to retinal vascular diseases treated with intravitreal dexamethasone or ranibizumab. Retina. 2016;36(12):2319-2328. doi:10.1097/IAE.0000000000001070

79. Hwang HS, Chae JB, Kim JY, Kim DY. Association between hyperreflective dots on spectral-domain optical coherence tomography in macular edema and response to treatment. Invest Ophthalmol Vis Sci. 2017;58(13):5958-5967. doi:10.1167/ iovs.17-22725
80. Schreur V, Altay L, van Asten F, et al. Hyperreflective foci on optical coherence tomography associate with treatment outcome for anti-VEGF in patients with diabetic macular edema. PLoS One. 2018;13(10):e0206482. doi:10.1371/journal.pone.0206482

81. Vujosevic $\mathrm{S}$, Torresin $\mathrm{T}$, Bini $\mathrm{S}$, et al. Imaging retinal inflammatory biomarkers after intravitreal steroid and anti-VEGF treatment in diabetic macular oedema. Acta Ophthalmol. 2017;95 (5):464-471. doi:10.1111/aos.13294

82. Sun JK, Lin MM, Lammer J, et al. Disorganization of the retinal inner layers as a predictor of visual acuity in eyes with center-involved diabetic macular edema. JAMA Ophthalmol. 2014;132(11):1309-1316. doi:10.1001/ jamaophthalmol. 2014.2350

83. Das R, Spence G, Hogg RE, Stevenson M, Chakravarthy U. Disorganization of inner retina and outer retinal morphology in diabetic macular edema. JAMA Ophthalmol. 2018;136 (2):202-208. doi:10.1001/jamaophthalmol.2017.6256

84. Radwan SH, Soliman AZ, Tokarev J, Zhang L, van Kuijk FJ, Koozekanani DD. Association of disorganization of retinal inner layers with vision after resolution of center-involved diabetic macular edema. JAMA Ophthalmol. 2015;133(7):820-825. doi:10.1001/jamaophthalmol.2015.0972

85. Zur D, Iglicki M, Sala-Puigdollers A, et al.; International Retina Group (IRG). Disorganization of retinal inner layers as a biomarker in patients with diabetic macular oedema treated with dexamethasone implant. Acta Ophthalmol. 2020;98(2): e217-e223. doi:10.1111/aos.14230.

86. Yanyali A, Bozkurt KT, Macin A, Horozoglu F, Nohutcu AF. Quantitative assessment of photoreceptor layer in eyes with resolved edema after pars plana vitrectomy with internal limiting membrane removal for diabetic macular edema. Ophthalmologica. 2011;226(2):57-63. doi:10.1159/000327597

87. Shin HJ, Lee SH, Chung H, Kim HC. Association between photoreceptor integrity and visual outcome in diabetic macular edema. Graefes Arch Clin Exp Ophthalmol. 2012;250(1):61-70. doi:10.1007/s00417-011-1774-x

88. Muftuoglu IK, Mendoza N, Gaber R, Alam M, You Q, Freeman WR. Integrity of outer retinal layers after resolution of central involved diabetic macular edema. Retina. 2017;37 (11):2015-2024. doi:10.1097/IAE.0000000000001459

89. Rangaraju L, Jiang X, McAnany JJ, et al. Association between visual acuity and retinal layer metrics in diabetics with and without macular edema. J Ophthalmol. 2018;2018:1089043.

90. Wong RL, Lee JW, Yau GS, Wong IY. Relationship between outer retinal layers thickness and visual acuity in diabetic macular edema. Biomed Res Int. 2015;2015:981471. doi:10.1155/2015/ 981471

91. Eliwa TF, Hussein MA, Zaki MA, Raslan OA. Outer retinal layer thickness as good visual predictor in patients with diabetic macular edema. Retina. 2018;38(4):805-811. doi:10.1097/ IAE.0000000000001599

92. Abd Elhamid AH. Quantitative assessment of outer retinal layer and photoreceptor outer segment layer and their relation to visual acuity in diabetic macular edema. $J$ Ophthalmol. 2019;2019:8216150. doi:10.1155/2019/8216150

93. Hatz K, Ebneter A, Tuerksever C, Pruente C, Zinkernagel M. Repeated dexamethasone intravitreal implant for the treatment of diabetic macular oedema unresponsive to anti-VEGF therapy: outcome and predictive SD-OCT features. Ophthalmologica. 2018;239(4):205-214. doi:10.1159/000485852

94. Castro-Navarro V, Monferrer-Adsuara C, Navarro-Palop C, Montero-Hernández J, Cervera-Taulet E. Effect of dexamethasone intravitreal implant on visual acuity and foveal photoreceptor integrity in macular edema secondary to retinal vascular disease. Ophthalmologica. 2021;244(1):83-92. doi:10.1159/000512195 
95. Jain A, Saxena S, Khanna VK, Shukla RK, Meyer CH. Status of serum VEGF and ICAM-1 and its association with external limiting membrane and inner segment-outer segment junction disruption in type 2 diabetes mellitus. Mol Vis. 2013;19:1760-1768.

96. De S, Saxena S, Kaur A, et al. Sequential restoration of external limiting membrane and ellipsoid zone after intravitreal anti-VEGF therapy in diabetic macular oedema. Eye. 2021;35 (5):1490-1495. doi:10.1038/s41433-020-1100-0

97. Bansal P, Agarwal A, Gupta V, Singh R, Gupta A. Spectral domain optical coherence tomography changes following intravitreal dexamethasone implant, Ozurdex ${ }^{\circledR}$ in patients with uveitic cystoid macular edema. Indian $J$ Ophthalmol. 2015;63 (5):416-422. doi:10.4103/0301-4738.159870

98. Altunel O, Duru N, Goktas A, Ozkose A, Goktas E, Atas M. Evaluation of foveal photoreceptor layer in eyes with macular edema associated with branch retinal vein occlusion after ozurdex treatment. Int Ophthalmol. 2019;2019(2):333-339. doi:10.1007/ s10792-016-0261-0

99. Iacono P, Parodi MB, Scaramuzzi M, Bandello F. Morphological and functional changes in recalcitrant diabetic macular oedema after intravitreal dexamethasone implant. $\mathrm{Br} J$ Ophthalmol. 2017;101(6):791-795. doi:10.1136/bjophthalmol-2016-308726

100. Bonfiglio V, Reibaldi M, Pizzo A, et al. Dexamethasone for unresponsive diabetic macular oedema: optical coherence tomography biomarkers. Acta Ophthalmol. 2019;97(4):e540-e544. doi:10.1111/aos.13935

101. Chen Y, Li J, Yan Y, Shen X. Diabetic macular morphology changes may occur in the early stage of diabetes. $B M C$ Ophthalmol. 2016;16:12. doi:10.1186/s12886-016-0186-4

102. Wanek J, Blair NP, Chau FY, Lim JI, Leiderman YI, Shahidi M. Alterations in retinal layer thickness and reflectance at different stages of diabetic retinopathy by en face optical coherence tomography. Invest Ophthalmol Vis Sci. 2016;57(9):OCT341347. doi:10.1167/iovs.15-18715
103. Huang XL, Song YP, Ding Q, Chen X, Hong L. Evaluation of outer retinal tubulations in diabetic macular edema underwent anti-VEGF treatment. Int $J$ Ophthalmol. 2019;12(3):442-450. doi:10.18240/ijo.2019.03.15

104. Fonollosa A, Zarranz-Ventura J, Valverde A, et al. Predictive capacity of baseline hyperreflective dots on the intravitreal dexamethasone implant $\left(\right.$ Ozurdex $\left.{ }^{\circledR}\right)$ outcomes in diabetic macular edema: a multicenter study. Graefes Arch Clin Exp Ophthalmol. 2019;257(11):2381-2390. doi:10.1007/s00417-019-04446-4

105. Lee H, Jang H, Choi YA, Kim HC, Chung H. Association between soluble CD14 in the aqueous humor and hyperreflective foci on optical coherence tomography in patients with diabetic macular edema. Invest Ophthalmol Vis Sci. 2018;59(2):715-721. doi:10.1167/iovs.17-23042

106. Jonas JB, Jonas RA, Neumaier M, Findeisen P. Cytokine concentration in aqueous humor of eyes with diabetic macular edema. Retina. 2012;32(10):2150-2157. doi:10.1097/IAE.0b01 3e3182576d07

107. Lafuma A, Brézin A, Lopatriello S, et al. Evaluation of non-medical costs associated with visual impairment in four European countries: France, Italy, Germany and the UK. Pharmacoeconomics. 2006;24(2):193-205. doi:10.2165/ 00019053-200624020-00007

108. Abraldes MJ, Pareja A, Roura M; En representación del grupo del estudio OBSERVAR. Analysis of costs associated with the management and morbidity of diabetic macular oedema and macular oedema secondary to retinal vein occlusion. Arch Soc Esp Oftalmol. 2016;91(6):273-280. doi:10.1016/j.oftal.2015.11.019

109. Romero-Aroca P, de la Riva-fernandez S, Valls-Mateu A, et al. Cost of diabetic retinopathy and macular oedema in a population, an eight year follow up. BMC Ophthalmol. 2016;16:136. doi:10.1186/s12886-016-0318-x
Clinical Ophthalmology

\section{Publish your work in this journal}

Clinical Ophthalmology is an international, peer-reviewed journal covering all subspecialties within ophthalmology. Key topics include: Optometry; Visual science; Pharmacology and drug therapy in eye diseases; Basic Sciences; Primary and Secondary eye care; Patient Safety and Quality of Care Improvements. This journal is indexed on PubMed

\section{Dovepress}

Central and CAS, and is the official journal of The Society of Clinical Ophthalmology (SCO). The manuscript management system is completely online and includes a very quick and fair peer-review system, which is all easy to use. Visit http://www.dovepress.com/ testimonials.php to read real quotes from published authors. 